\title{
Automatic Aorta Segmentation and Valve Landmark Detection in C-Arm CT: Application to Aortic Valve Implantation
}

\author{
Yefeng Zheng ${ }^{1}$, Matthias John' ${ }^{2}$, Rui Liao ${ }^{1}$, Jan Boese ${ }^{2}$, Uwe Kirschstein ${ }^{2}$, \\ Bogdan Georgescu ${ }^{1}$, S. Kevin Zhou ${ }^{1}$, Jörg Kempfert ${ }^{3}$, \\ Thomas Walther ${ }^{4}$, Gernot Brockmann ${ }^{5}$, and Dorin Comaniciu ${ }^{1}$ \\ 1 Siemens Corporate Research, Princeton, USA \\ 2 Siemens AG, Healthcare Sector, Forchheim, Germany \\ 3 Department of Cardiac Surgery, Heart Center, University of Leipzig, Germany \\ 4 Department of Cardiac Surgery, Kerckoff Heart Center, Bad Nauheim, Germany \\ 5 Department of Cardiovascular Surgery, German Heart Center, Munich, Germany \\ yefeng.zheng@siemens. com
}

\begin{abstract}
C-arm CT is an emerging imaging technique in transcatheter aortic valve implantation (TAVI) surgery. Automatic aorta segmentation and valve landmark detection in a C-arm CT volume has important applications in TAVI by providing valuable 3D measurements for surgery planning. Overlaying 3D segmentation onto $2 \mathrm{D}$ real time fluoroscopic images also provides critical visual guidance during the surgery. In this paper, we present a part-based aorta segmentation approach, which can handle aorta structure variation in case that the aortic arch and descending aorta are missing in the volume. The whole aorta model is split into four parts: aortic root, ascending aorta, aortic arch, and descending aorta. Discriminative learning is applied to train a detector for each part separately to exploit the rich domain knowledge embedded in an expertannotated dataset. Eight important aortic valve landmarks (three aortic hinge points, three commissure points, and two coronary ostia) are also detected automatically in our system. Under the guidance of the detected landmarks, the physicians can deploy the prosthetic valve properly. Our approach is robust under variations of contrast agent. Taking about 1.4 seconds to process one volume, it is also computationally efficient.
\end{abstract}

\section{Introduction}

Affecting $1.8 \%$ of the global population and $10.7 \%$ of persons older than 65 , aortic valve disease is the most common valvular disease in developed countries [1. Implantation of a prosthetic aortic valve is often necessary to replace the severely damaged native valve. Though open-chest valve surgery is a well established procedure, minimally invasive transcatheter aortic valve implantation is an emerging technique, especially for high-risk patients, to minimize the surgical trauma. Before the surgery, several important parameters of the aortic valve (as shown in Fig. 1) need to be extracted for surgery planning. For example, the diameter of aortic valve annulus needs to be measured to select a 

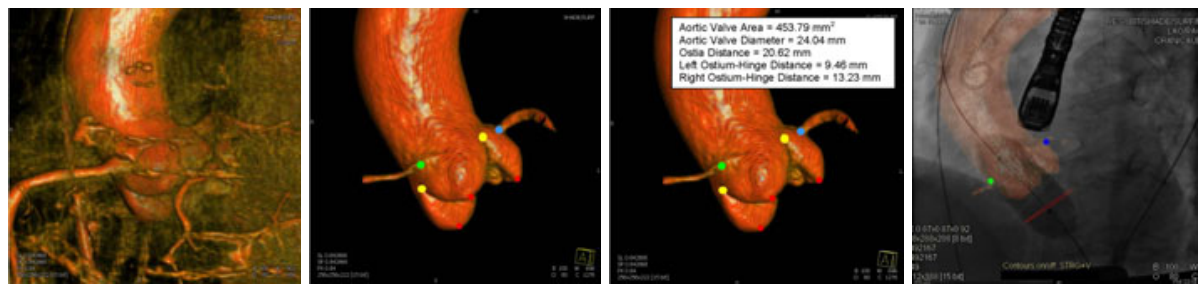

Fig. 1. Applications of C-arm CT to transcatheter aortic valve implantation. Left: A C-arm CT volume. Left Middle: Automatically segmented aorta together with the detected valve landmarks. Right Middle: 3D geometric measurements of the valve. Right: Overlay of the segmented aorta onto a 2D fluoroscopic image for visual guidance during surgery.

prosthetic valve with an appropriate size. During the surgery, 2D fluoroscopic images are captured in real time in a $\mathrm{C}$-arm system to provide guidance to physicians 2. The aortic root structure is distinguishable from the background only during a short period when the contrast agent is applied. However, the contrast agent is toxic and its usage should be minimized. Computed tomography (CT) is often used to provide the necessary 3D geometric measurements in surgery planning. However, CT is rarely used during valve implantation surgery because $2 \mathrm{D} / 3 \mathrm{D}$ overlay (or registration) of data captured from different imaging devices is quite difficult. Recently, C-arm CT emerges as a new imaging technique with the following advantages, compared to conventional CT. Since both the 3D volume and 2D fluoroscopic images are captured on the same device within a short time interval, overlay of the $3 \mathrm{D}$ patient-specific aorta model onto a $2 \mathrm{D}$ image is straightforward and accurate (except patient motion). Besides providing visual guidance, the extracted aortic root can predict the best $\mathrm{C}$-arm angulation (the optimal orientation of the imaging plane) to mitigate the foreshortening effect. For more details on the clinical applications of C-arm CT, please refer to [23].

A fully automatic system of aorta segmentation and valve landmark detection pays a key role in seamlessly integrating C-arm CT into the TAVI workflow. There are only a few methods proposed in literature to segment the aorta in a 3D volume. Zhao et al. 4] proposed a semi-automatic method to segment aorta in MR images. A user needs to manually select a seed point to initialize the fast marching method, which generates a rough segmentation result. Graph cut is exploited for final boundary refinement. Rueckert et al. [5] presented a tracking based segmentation method for the ascending and descending aortas. The intersection of the ascending/descending aorta with the image slice is roughly a circle, which is easy to track along slices. However, the curved aortic arch cannot be handled elegantly. Since the aorta is a tubular structure, many generic tubular structure detection approaches 6 6/7/8 can be extended to detect and segment it. Automatic aortic valve landmark detection is a new topic with very few publications in literature. Ionasec et al. [9] presented a comprehensive aortic valve model, which included the important valve landmarks, e.g., hinge points, commissure points, and coronary ostia. All the previous work focuses on a 
relatively consistent imaging protocol with much fewer variations than ours. For example, a roughly same portion of the aorta is captured in the volume and the usage of contrast agent is consistent, resulting in stable image characteristics. Furthermore, most of the previous approaches are semi-automatic (a user needs to click at least one point [4]) and very slow (taking up to 450 seconds to process one volume [7]).

All of the previous methods work on the well established imaging modalities, such as MR and CT. However, automatic segmentation of the aorta in a C-arm CT volume is far more challenging. First, the image quality from different clinical sites varies quite a lot since $\mathrm{C}$-arm $\mathrm{CT}$ is too new to have a well accepted scanning protocol. We also observed significant variations inside the same clinical site since physicians were testing different scanning parameters (e.g., the amount of contrast agent and timing of the image acquisition). Conventional image processing techniques, e.g., intensity-based thresholding, region growing, and the watershed method, are not robust under such large variations. We propose to use machine learning techniques to exploit the rich information embedded in an expert-annotated dataset. Second, the field of view varies quite a lot for a C-arm CT volume. For example, the aortic arch and descending aorta may be captured in some volumes (see the first two examples in Fig. 2), but missing in others (see the last two examples in Fig. 2). To address this challenge, we propose a part-based aorta model. As shown in Fig. 3, the whole aorta is split into four parts: aortic root, ascending aorta, aortic arch, and descending aorta. Using the part-based model, the whole aorta does not need to be fully present.
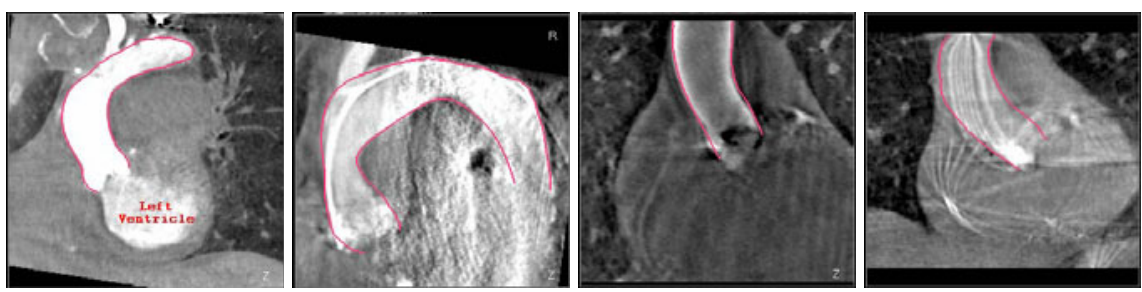

Fig. 2. Automatic aorta segmentation on a few example volumes. Left: Good contrast, however, with severe valve regurgitation. Left Middle: Fair image quality. Right Middle: Contrast agent is almost washed out due to bad timing. Right: Streak artifacts generated by the catheters.

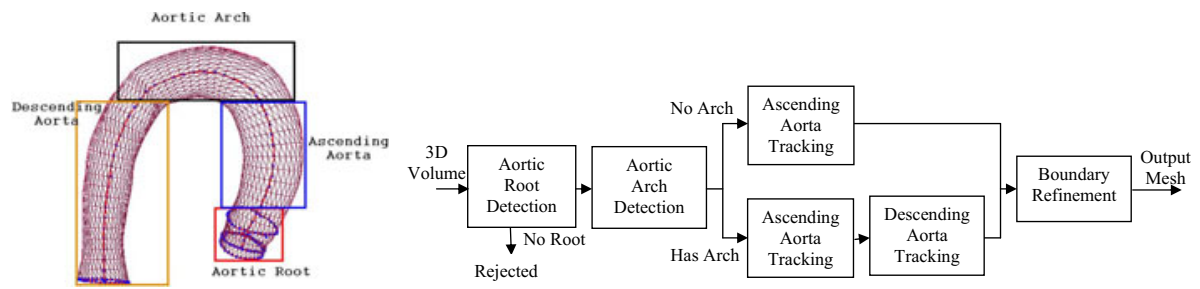

Fig. 3. Part-based aorta model (left) and automatic segmentation workflow (right) 
Depending on the structure that can be detected, different workflows can be exploited, therefore, a large structure variation can be handled elegantly.

\section{Part-Based Aorta Modeling and Segmentation}

Due to the variation in the field of view, the aorta captured in a C-arm CT volume changes a lot in its structure. In this paper, we present a part-based aorta model (as shown in Fig. 3) by splitting the whole aorta into four parts: aortic root, ascending aorta, aortic arch, and descending aorta. The aortic root is required to be present in this application, therefore, it is detected and segmented as the first step. To be specific, we use the recently proposed marginal space learning (MSL) method [10] to segment the aortic root. MSL is an efficient method to detect and segment a 3D anatomical structure in medical images based on a discriminative machine learning technique. It is robust and works for different imaging modalities. Due to the space limit, we would like to refer readers to 10 for more details of MSL. As shown in the system diagram in Fig. 3 , the aortic root is detected first. If no aortic root is detected, the input volume is rejected. We then detect the aortic arch. Similarly, MSL is exploited to train a separate detector for the aortic arch. For about half of the volumes in our dataset, the aortic arch may be out of the field of view. If no aortic arch is present, normally the descending aorta is also missing in the volume (see the last two cases in Fig. 2).

The length of the ascending and descending aortas captured in a volume varies significantly. It is difficult to detect them as whole objects. We propose to use a tracking technique to deal with this variation. Since the intersection of the ascending and descending aortas with an image slice is close to a circle, we train a 2D circle detector using Haar wavelet features and the boosting learning algorithm [10] to detect aortic circles as primitive structures for tracking. Starting from the aortic root, we detect an aortic circle on the next slice (toward the patient's head). The detector outputs multiple circle candidates around the true position. We pick the one closest to the circle on the current slice. If the aortic arch is detected in the volume, the tracking procedure stops on the slice touching the aortic arch. Otherwise, it stops when no aortic circle is detected or it reaches the top volume border. Tracking of the descending aorta is similar except that it starts from the aortic arch and moves toward the patient's toe. It stops on the slice with no aortic circle detected.

Assembling all the aortic parts together (the tracked aortic circles, aortic root, and aortic arch if it is present), we get an initial surface mesh of the aorta. The initialization is close to the true aorta boundary, however, a circle does not fit the boundary exactly. A learning based boundary detector is applied for final boundary delineation. Specifically, a two-step iterative approach is used. 1) Use the learning-based boundary detector to adjust each mesh point along the surface normal to the optimal position where the response of the boundary detector is the largest. 2) Apply generic mesh smoothing [11] to get a smooth surface. The above two steps repeat a few iterations to improve the boundary delineation accuracy. 
MSL [10] can efficiently detect an object as a whole. However, it cannot deal with structural variations. Therefore, almost all previous work uses bottomup approaches 45678 to track the aorta centerline to handle the variations. They are neither automatic nor robust on noisy images. In comparison, we use MSL to detect the aortic root and arch, and use bottom-up tracking to detect ascending/descending aortas that have large variations in length. Our system is a nice combination of both approaches.

\section{Aortic Valve Landmark Detection}

Besides segmenting the aorta, we also detect eight aortic valve landmarks: three aortic hinge points, three aortic commissure points, and left and right coronary ostia since they are important in both surgery planning and providing visual guidance during surgery 3. Though it is possible to detect each landmark independently, the detection results may be inconsistent in geometry. It also wastes computation power by ignoring the strong geometric constraint among the landmarks. We propose to use a hierarchical approach by first detecting a global object comprised with all eight valve landmarks. From the position, orientation, and scale of this global object, we can infer the rough position of individual landmarks. Each landmark is then refined in a small region (e.g., a cube of $20 \mathrm{~mm}$ centered on the initial position) under the guidance of its own specific landmark detector.

Similar to the aortic root detection, we use marginal space learning (MSL) [10] to efficiently detect the position, orientation, and scales of the global landmark object. For a learning based method, we need to specify the ground truth of object pose for each training volume, therefore, a learning algorithm can learn the implicit relationship to infer the correct pose from an unseen volume. However, there is no standard way to define the pose of the global object containing eight landmarks. After detecting the global landmark object, we align the mean shape (which is the average shape of the training set after global transformation has been compensated) to the global pose to get an initial estimate of each individual landmark's position (see Eq. (2) ). In this paper, we propose a method to search for an optimal shape which can represent the whole shape population accurately, therefore improving the initialization accuracy of the landmarks. Given a group of shapes, $M_{1}, M_{2}, \ldots, M_{N}$, we want to find an optimal shape $\bar{m}$ to represent the whole population such that it can minimize the residual errors after alignment,

$$
\bar{m}=\arg \min _{m} \sum_{i=1}^{N}\left\|\mathcal{T}_{i}(m)-M_{i}\right\|^{2} .
$$

The optimal shape $\bar{m}$ is called the mean shape in this paper. $\mathcal{T}_{i}$ is the corresponding transformation from the mean shape $\bar{m}$ to each individual shape $M_{i}$. This procedure is called generalized Procrustes analysis in statistical shape analysis [12]. 
Previously, the generalized Procrustes analysis is only performed under the similarity transformation (i.e., $\mathcal{T}$ is a similarity transformation). MSL can estimate anisotropic scales quite efficiently. With more deformation compensated, the mean shape is more accurate to represent the whole shape population. Therefore, in our approach $\mathcal{T}$ represents translation $\left(T=[X, Y, Z]^{\prime}\right)$, rotation (represented as a rotation matrix $R$ ), and anisotropic scaling $\left(S_{x}, S_{y}, S_{z}\right)$. The transformation of a $3 \mathrm{D}$ point $P$ is

$$
\mathcal{T}(P)=R\left[\begin{array}{ccc}
S_{x} & 0 & 0 \\
0 & S_{y} & 0 \\
0 & 0 & S_{z}
\end{array}\right] P+T .
$$

To the best of our knowledge, there are no closed-form solutions for estimating the anisotropic similarity transformation. We propose an iterative algorithm. We first estimate the similarity transformation (translation, rotation, and isotropic scaling), which has closed-form solutions [12]. After compensating the similarity transformation, we estimate the three anisotropic scaling parameters $\left(S_{x}, S_{y}\right.$, $S_{z}$ ), for which we derive a closed-form solution. With a module solving the anisotropic similarity transformation between two shapes, we can plug it into the generalized Procrustes analysis method to search for the optimal mean shape $\bar{m}$. Besides the optimal mean shape, the transformation $\mathcal{T}_{i}$ of the mean shape to each example shape $M_{i}$ is also calculated as a by-product, which provides the pose ground truth that MSL can learn to estimate.

\section{Experiments}

A dataset of $192 \mathrm{C}$-arm CT volumes from 152 patients were collected from two clinical sites to evaluate our method. The size of each slice in a volume is $256 \times 256$ or $512 \times 512$ pixels. A volume contains around $200-300$ slices. The image resolution is isotropic and varies from volume to volume in the range of $[0.70,0.84] \mathrm{mm}$.

A four-fold cross-validation is performed to evaluate our algorithm. The aorta segmentation accuracy is measured using the symmetric point-to-mesh distance $E_{p 2 m}[10$. The mean segmentation error of the aorta is $1.1 \mathrm{~mm}$, with a standard deviation of $0.41 \mathrm{~mm}$. We cannot compare our error with those reported in the literature directly because they used different datasets captured from different imaging modalities. Roughly, our accuracy is comparable to (or better than) the state-of-the-art, e.g., $1.55 \mathrm{~mm}$ mean error reported in 4 on $21 \mathrm{MR}$ datasets and $1.4 \mathrm{~mm}$ mean error reported in [7] on $23 \mathrm{CT}$ datasets. Fig. 2] shows aorta segmentation results on a few volumes. The first example shows a volume with good image quality. However, due to severe valve regurgitation the contrast leaks into the left ventricle. The third example shows a case with bad image acquisition timing where the contrast agent has almost been washed out. Severe streak artifacts generated by catheters are clearly visible in the last volume.

In the following experiment, we evaluate the valve landmark detection accuracy. There are a total of 28 volumes with extremely poor image quality that 
the landmarks cannot be identified even by an expert (though the aorta can be successfully segmented from these volumes). So these images are excluded. A four-fold cross-validation is performed on the remaining 164 volumes for aortic valve landmark detection. The landmark detection accuracy is measured using the Euclidean distance from the detected landmark to the ground truth. Table 1 shows the detection errors. After global landmark object pose estimation, we can get a good initial estimate of the landmark position. The mean errors range from 5.40 to $5.81 \mathrm{~mm}$ for different landmarks. After local refinement for each landmark, the error is further reduced. For example, the mean error for the aortic hinges reduces from $5.40 \mathrm{~mm}$ to $2.41 \mathrm{~mm}$. Fig. 4 shows the detected valve landmarks in two typical volumes. Our approach is computationally efficient, taking about 1.4 seconds to process a volume on a computer with $3.2 \mathrm{GHz}$ duo-core processors and 3 GB memory. It is at least 10 times faster than the previous methods [5]7.

Table 1. Aortic valve landmark detection errors based on a four-fold cross-validation on 164 volumes. The mean, standard deviation (STD), and median of the errors (measured in millimeters) are reported.

\begin{tabular}{|c|c|c|c|c|c|c|c|}
\hline & \begin{tabular}{|c|}
\multicolumn{2}{|c|}{ Aortic H } \\
Mean|STD
\end{tabular} & $\frac{\text { nges }}{\text { Median }}$ & \begin{tabular}{|c|}
\multicolumn{2}{|c|}{ Coronary } \\
Sean \\
\end{tabular} & $\begin{array}{l}\text { Ostia } \\
\text { Median }\end{array}$ & \begin{tabular}{|l|} 
Aortic \\
Mean \\
\end{tabular} & ISTD & $\frac{m \text { missures }}{\text { Median }}$ \\
\hline$\overline{\text { After Global Pose Estimation }}$ & \begin{tabular}{|l|l|}
5.40 & 2.51 \\
\end{tabular} & 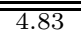 & \begin{tabular}{|l|l|}
5.81 & 2.45 \\
\end{tabular} & 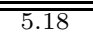 & 5.43 & 2.26 & 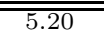 \\
\hline After Local Refinement & \begin{tabular}{l|l}
2.41 & 1.50 \\
\end{tabular} & 1.90 & \begin{tabular}{|l|l|}
2.74 & 2.43 \\
\end{tabular} & 1.77 & 3.46 & 1.78 & 3.11 \\
\hline
\end{tabular}
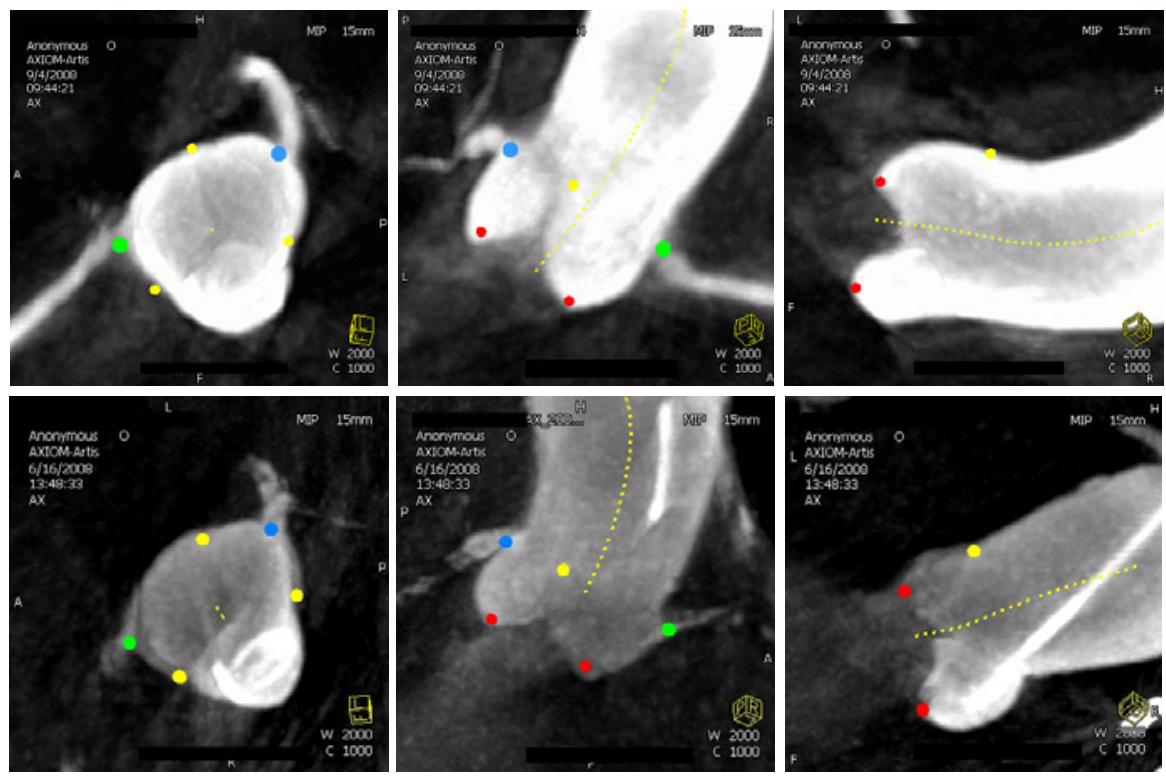

Fig. 4. The aortic valve landmark detection results on two example datasets with red dots for the hinge points, yellow for the commissure points, blue for the left coronary ostium, and green for the right coronary ostium. Each row shows three orthogonal cuts of a volume. 


\section{Conclusion}

In this paper, we presented a fully automatic aorta segmentation and valve landmark detection system in C-arm CT with applications to transcatheter aortic valve implantation (TAVI). The initial clinical trial demonstrated the usefulness of our system in the TAVI workflow, e.g., providing a proper angulation to avoid large tilting of a prosthetic valve after deployment [3. Our approach is generic, therefore can be extended easily to other imaging modalities by simple retraining, without any manual parameter tuning. A similar system has been built on cardiac $\mathrm{CT}$ datasets to use conventional $\mathrm{CT}$ for surgery planning.

\section{References}

1. Nkomo, V.T., Gardin, J.M., Skelton, T.N., Gottdiener, J.S., Scott, C.G., Enriquez-Sarano, M.: Burden of valvular heart diseases: a population-based study. Lancet 368(10), 1005-1011 (2006)

2. Gessat, M., Merk, D.R., Falk, V., Walther, T., Jacobs, S., Nöttling, A., Burgert, O.: A planning system for transapical aortic valve implantation. In: Proc. of SPIE Medical Imaging (2009)

3. John, M., Liao, R., Zheng, Y., Nöttling, A., Boese, J., Kirschstein, U., Kempfert, J., Walther, T.: System to guide transcatheter aortic valve implantations based on interventional 3D C-arm CT imaging. In: Proc. Int'l. Conf. Medical Image Computing and Computer Assisted Intervention (2010)

4. Zhao, F., Zhang, H., Wahle, A., Scholz, T.D., Sonka, M.: Automated 4D segmentation of aortic magnetic resonance images. In: Proc. British Machine Vision Conference (2006)

5. Rueckert, D., Burger, P., Forbat, S.M., Mohiaddin, R.D., Yang, G.Z.: Automatic tracking of the aorta in cardiovascular MR images using deformable models. IEEE Trans. Medical Imaging 16(5), 581-590 (1997)

6. Behrens, T., Rohr, K., Stiehl, H.H.: Robust segmentation of tubular structures in 3-D medical images by parametric object detection and tracking. IEEE Trans. Syst., Man, Cybern. B 33(14), 554-561 (2003)

7. de Bruijne, M., van Ginneken, B., Viergever, M.A., Niessen, W.J.: Adapting active shape models for 3D segmentation of tubular structures in medical images. In: Proc. Information Processing in Medical Imaging, pp. 136-147 (2003)

8. Saur, S.C., Kühnel, C., Boskamp, T., Székely, G., Cattin, P.: Automatic ascending aorta detection in CTA datasets. In: Bildverarbeitung für der Medizin 2008, pp. 323-327 (2008)

9. Ionasec, R., Georgescu, B., Gassner, E., Vogt, S., Kutter, O., Scheuering, M., Navab, N., Comaniciu, D.: Dynamic model-driven quantification and visual evaluation of the aortic valve from 4D CT. In: Proc. Int'l. Conf. Medical Image Computing and Computer Assisted Intervention, pp. 686-694 (2008)

10. Zheng, Y., Barbu, A., Georgescu, B., Scheuering, M., Comaniciu, D.: Four-chamber heart modeling and automatic segmentation for 3D cardiac CT volumes using marginal space learning and steerable features. IEEE Trans. Medical Imaging 27(11), 1668-1681 (2008)

11. Taubin, G.: Optimal surface smoothing as filter design. In: Proc. European Conf. Computer Vision, pp. 283-292 (1996)

12. Dryden, I.L., Mardia, K.V.: Statistical Shape Analysis. John Wiley, Chichester (1998) 